\title{
Vascular mimicry by VE-cadherin enables trophoblast endovascular invasion and spiral artery remodeling during placental development
}

Derek C. Sung ${ }^{1}$, Xiaowen Chen ${ }^{1}$, Mei Chen ${ }^{1}$, Jisheng Yang ${ }^{1}$, Susan Schultz ${ }^{2}$, Apoorva Babu, Yitian Xu' ${ }^{1}$, Siqi Gao ${ }^{1}$, TC Stevenson Keller IV ${ }^{1}$, Patricia Mericko ${ }^{1}$, Michelle Lee ${ }^{3}$, Ying Yang ${ }^{4}$, Joshua P. Scallan ${ }^{4}$, and Mark L. Kahn ${ }^{1 *}$

${ }^{1}$ Cardiovascular Institute, Department of Medicine, Perelman School of Medicine, University of Pennsylvania, Philadelphia, Pennsylvania

${ }^{2}$ Department of Radiology, Hospital of the University of Pennsylvania, Philadelphia, Pennsylvania

${ }^{3}$ University Laboratory Animal Resources, University of Pennsylvania, Philadelphia, Pennsylvania

${ }^{4}$ Department of Molecular Pharmacology and Physiology, University of South Florida, Tampa, Florida

${ }^{*}$ Corresponding author

Please address correspondence to:

Dr. Mark L. Kahn, MD

Department of Medicine and Cardiovascular Research Institute

Smilow Center for Translational Research Room 11-123

3400 Civic Center Blvd

Philadelphia, PA 19104

Telephone: 215-898-9007

E-mail: markkahn@pennmedicine.upenn.edu

Keywords: placenta, trophoblasts, CDH5, VE-cadherin, vascular mimicry, preeclampsia 


\section{Abstract}

2 During formation of the mammalian placenta trophoblasts invade the maternal decidua and

3 remodel spiral arteries to bring maternal blood into the placenta. This process, known as

4 endovascular invasion, is thought to involve the adoption of functional characteristics of vascular

5 endothelial cells (ECs) by trophoblasts through a process termed vascular mimicry. The genetic

6 and molecular basis of vascular mimicry remains poorly defined, however, and whether

7 trophoblasts utilize specialized endothelial proteins in an analogous manner to create vascular

8 channels remains untested. Vascular endothelial (VE-)cadherin is a homotypic adhesion protein

9 that is expressed selectively by ECs in which it enables formation of tight vessels and regulation

10 of EC junctions. VE-cadherin is also expressed in invasive trophoblasts and is a prime

11 candidate for a molecular mechanism of vascular mimicry by those cells. Here, we show that

12 the VE-cadherin is required for trophoblast migration and endovascular invasion into the

13 maternal decidua in the mouse. VE-cadherin deficiency results in loss of spiral artery

14 remodeling that leads to decreased flow of maternal blood into the placenta, fetal growth

15 restriction, and death. These studies identify a non-endothelial role for VE-cadherin in

16 trophoblasts during placental development and suggest that endothelial proteins may play

17 functionally unique roles in trophoblasts that do not simply mimic those in ECs. 


\section{Introduction}

During placental development in mice and humans, fetal trophoblasts invade the maternal decidua by a process known as endovascular invasion to remodel and connect to

21 maternal spiral arteries ${ }^{1-3}$. This connection allows the flow of maternal blood through

22 trophoblast-lined sinuses characteristic of hemochorial placentation ${ }^{4,5}$. Shallow trophoblast

23 invasion, deficient spiral artery (SA) remodeling, and poor remodeling of the maternal decidua

24 are features of placental dysfunction such as preeclampsia, a hypertensive condition of

25 pregnancy that can lead to maternal and fetal complications ${ }^{6,7}$. Despite the broad and clinically

26 significant impacts of placental dysfunction, the mechanisms controlling trophoblast

27 endovascular invasion and SA remodeling remain poorly defined in vivo.

Invasive trophoblasts are believed to adopt an endothelial-like state by expressing endothelial specific genes ${ }^{8-10}$, a process that has been termed 'vascular mimicry' (reviewed in ${ }^{5}$ ). Invasive trophoblasts in human and mouse placentas express vascular endothelial (VE)cadherin (gene name Cdh5) during remodeling of SAs ${ }^{9-11}$. Invasive trophoblasts in preeclamptic placentas lack VE-cadherin ${ }^{11}$, and loss of VE-cadherin reduces trophoblast invasion in vitro ${ }^{12}$. These studies suggest a functional role for VE-cadherin in endovascular invasion and vessel formation. VE-cadherin is a well-studied cell-cell adhesion protein in the vascular endothelium where it regulates vascular integrity and growth and endothelial barrier function ${ }^{13-15}$. In vitro studies have suggested that VE-cadherin may regulate trophoblast-endothelial interactions ${ }^{16}$, but the requirement for VE-cadherin in trophoblasts during placental development in vivo remains unknown.

In the present study, we functionally tested the role of VE-cadherin in trophoblasts during placental development in mice. We find that conditional deletion of VE-cadherin from trophoblasts disrupts trophoblast invasion into the decidua and SA remodeling, resulting in placental insufficiency and fetal growth restriction. We show that VE-cadherin is important for

43 trophoblasts to interact with and displace SA endothelium. These studies identify a molecular

44 mechanism by which fetal trophoblasts use VE-cadherin to invade and remodel the maternal

45 environment for successful pregnancy that is relevant to preeclampsia pathogenesis. They also

46 provide a first functional test of the concept of trophoblast vascular mimicry in vivo, and suggest

47 that canonical vascular endothelial proteins may be used by vascular trophoblasts in the

48 placenta in ways that are specific for their function and do not merely mimic endothelial cell use. 
Results

Trophoblast-specific deletion of VE-cadherin restricts placental and fetal growth and causes embryonic lethality

To understand the role of VE-cadherin in trophoblasts we generated Cyp $19^{\mathrm{Cre}} ; \mathrm{Cdh} 5^{\mathrm{flft}}$ ('Cdh5 knockout') placentas and mice in which VE-cadherin (encoded by Cdh5) is deleted specifically in fetal trophoblasts. Immunostaining for VE-cadherin and the trophoblast marker CK8 demonstrated efficient deletion of VE-cadherin in trophoblasts but not ECs in the Cdh5 knockout placenta (Figure 1-Figure Supplement 1). Cdh5 knockout embryos were present at the expected Mendelian ratio at E10.5-12.5 (Figure 1-Table Supplement 1, $\mathrm{P}=0.7667$ ). However, there was an almost complete loss of trophoblast Cdh5 knockout embryos at E14.5E16.5 and postnatal day 21 (Figure 1-Table Supplement 1, $P<0.05$ and $P<0.005$, respectively). Examination of placentas at E12.5 revealed that trophoblast Cdh5 knockout placentas were smaller and paler than those of control littermates in the same uterus (Figure 1A-C). Cdh5 knockout embryos exhibited marked fetal growth restriction and hemorrhaging at E12.5 (green arrowheads, Figure 1D-F). Histological and immunofluorescence analysis of E12.5 knockout embryos showed growth defects in numerous organs, including the heart, brain, and liver (Figure 1-Figure Supplement 2). These data demonstrate that trophoblast-specific loss of VE-cadherin confers fetal growth restriction and lethality. Importantly, since Cyp19 ${ }^{\text {Cre }}$ activity is present only in placental trophoblasts ${ }^{17,18}$, these embryonic defects are secondary to placental defects.

Loss of trophoblast VE-cadherin disrupts endovascular invasion but not formation of the fetal vasculature

To characterize the placental defects conferred by trophoblast loss of VE-cadherin we performed histological staining with $\mathrm{H} \& \mathrm{E}$ and immunofluorescence staining for CK8 (trophoblasts), Endomucin (endothelial cells), and TER119 (red blood cells) on serial control and Cdh5 knockout placenta sections (Figure 2A-D). H\&E and immunofluorescence staining both showed abundant trophoblasts, marked by CK8 positivity, surrounding Endomucin ${ }^{+} \mathrm{SAs}$ within the decidua in control placentas (red arrowheads, Figure 2B, B'). Notably, trophoblasts surrounding maternal SAs were absent in knockout placentas (Figure 2D, D'), and quantification of number of trophoblasts and invasion depth into the decidua showed fewer and shallower invasion of trophoblasts overall (decidua, Figure 2E, F).

82

The findings described above suggested that Cdh5 knockout placentas were less able to carry maternal blood to nourish the growing embryo. Fetal and maternal RBCs can be 
83 differentiated by the presence of nuclei in fetal RBCs. The labyrinth in knockout placentas had

84 fewer enucleated TER119+ RBCs, indicating less maternal blood and consistent with paler

85 placentas observed by gross examination (white arrowheads, Figure 2B" vs. D"). To determine

86 whether loss of VE-cadherin affects the fetal placental vasculature, we quantified Endomucin ${ }^{+}$

87 vascular area in the labyrinth. We detected no differences in Endomucin ${ }^{+}$staining,

88 demonstrating that loss of VE-cadherin from trophoblasts does not disrupt formation of the fetal

89 placental capillary plexus (Figure 2B, D, G). These findings suggest that VE-cadherin is

90 important for trophoblast migration and for the association with SAs required to channel

91 maternal blood into the placenta.

\section{Loss of trophoblast VE-cadherin blocks displacement of spiral artery endothelial cells} and spiral artery remodeling

A critical early step in establishing maternal circulation to the placenta is trophoblast invasion of the decidua and its SAs. The observation that there were fewer VE-cadherindeficient trophoblasts adjacent to SAs and decreased maternal blood within the labyrinth suggested that trophoblast VE-cadherin may play a requisite role in SA remodeling. Since loss of vascular smooth muscle is a key step in SA remodeling, we stained control and knockout placentas for alpha-smooth muscle actin ( $\alpha$ SMA). SAs in Cdh5 knockout placentas exhibited persistent vascular smooth muscle coverage compared with those in control placentas (white

102 arrowheads, Figure 3A, B). These studies reveal that loss of trophoblast VE-cadherin disrupts

103 SA remodeling, which likely contributes to reduced maternal blood within the placenta.

During the process of SA remodeling, invasive trophoblasts displace the endothelial layer of SAs to direct maternal blood flow through trophoblast-lined sinuses into the labyrinth. In vitro studies have suggested that trophoblast expression of VE-cadherin may enable these cells

107 to adhere to SA endothelial cells $(E C s)^{16}$. The finding that trophoblast Cdh5 knockout placentas

108 fail to remodel SAs suggested that there may be defects in trophoblast-SA interactions. To

109 address the role of VE-cadherin at the site of trophoblast-SA connection, we first sought to

110 image the site at which trophoblasts connect to SAs. Close inspection of this region in control

111 placentas revealed a clear demarcation from luminal Endomucin ${ }^{+} \mathrm{SA}$ endothelium to luminal

$112 \mathrm{CK}^{+}$trophoblasts (white arrowheads, Figure 3C, D). In contrast, we found that SAs in VE-

113 cadherin knockout placentas maintained a layer of intact ECs despite being surrounded by

114 trophoblasts (white arrowheads, Figure 3C, D). Quantification of the percent of trophoblasts

115 immediately in contact with the lumen demonstrated that knockout placentas had a lower

116 percentage of trophoblasts and higher percentage of ECs covering the lumen compared to 
117 controls (Figure 3E). Together, these data suggest that VE-cadherin is required for trophoblast

118 displacement of spiral artery ECs during endovascular invasion for efficient maternal-fetal

119 circulatory connection.

Defective trophoblast invasion and spiral artery remodeling cause placental insufficiency and fetal distress

123 The finding that Cdh5 knockout placentas have less maternal blood and are associated

124 with mid-gestation embryonic lethality suggested that failed SA remodeling restricts maternal

125 blood flow into the placenta, thus causing fetal demise. Human placental insufficiency is

126 typically assessed with ultrasound measurement of placental hemodynamics and fetal heart

127 rate, a readout for overall fetal health. We therefore utilized Doppler ultrasound to measure peak

128 systolic and end diastolic velocities in the umbilical arteries (Figure 4A) and calculated

129 resistance and pulsatility indices (RI and $\mathrm{PI}$ ) and fetal heart rates to assess placental vascular

130 resistance and fetal wellbeing in individual concepti. Elevated RI and PI values are clinical

131 indicators of placental insufficiency in humans and are associated with conditions such as

132 preeclampsia and fetal growth restriction. Fetal heart rate is used as a clinical parameter for

133 fetal wellbeing, with fetal bradycardia indicative of fetal distress. While control embryos had RIs,

134 Pls, and fetal heart rates within range of previously published studies ${ }^{19}$, we found that

135 trophoblast Cdh5 knockout embryos exhibited significantly increased RI and PI (Figure 4B-D)

136 and significantly reduced fetal heart rates (Figure 4B, E), consistent with placental insufficiency

137 and fetal distress. Knockout embryos also exhibited reversal of end-diastolic flow as shown by

138 the directional change of velocity from negative (peak systole) to positive (end of diastole)

139 (Figure 4B), indicative of high vascular resistance. These hemodynamic data demonstrate

140 placental insufficiency that contributes to fetal growth restriction and fetal demise following loss

141 of trophoblast invasion and spiral artery remodeling. 


\section{Discussion}

143 The invasion of the maternal decidua by trophoblasts and their fusion to maternal SAs is

144 a critical step in establishing placental circulation. However, the mechanisms by which

145 trophoblast migration and endovascular invasion are accomplished remain largely unknown.

146 Vascular mimicry describes a model in which trophoblasts express endothelial molecular and

147 genetic programs ${ }^{4}$. However the exact function of specific endothelial genes in trophoblasts has

148 not been functionally assessed in vivo, and this model remains untested. In blood and lymphatic

149 ECs, VE-cadherin is used to maintain vascular integrity ${ }^{13-15}$, restrict endothelial migration ${ }^{14,20}$,

150 and regulate angiogenic growth ${ }^{21}$. While it is an attractive concept that trophoblasts may form

151 vascular sinuses using similar genetic programs, the findings that loss of VE-cadherin

152 decreases trophoblast cell migration and prevents SA remodeling suggests that trophoblasts

153 utilize VE-cadherin in a manner distinct from ECs. Our first genetic test of vascular mimicry in

154 the placenta suggests that the use of endothelial proteins by trophoblasts may be relatively

155 specific to their role in the placenta and not a simple reflection of vascular EC function.

In humans, defective SA remodeling and shallow trophoblast invasion are hallmarks of preeclampsia. Preeclampsia is a complex and heterogeneous disease with maternal and fetal contributions to its pathogenesis, and many in vitro models fail to fully recapitulate many

159 aspects of its pathophysiology. Most rodent models of preeclampsia utilize maternal genetic or 160 pharmacologic perturbations ${ }^{22,23}$, and there have been few in vivo models in which preeclamptic

161 features are recapitulated with fetal modulation of trophoblasts. Previous studies of human

162 placentas showed that invasive trophoblasts in severely preeclamptic placentas exhibit reduced

163 expression of VE-cadherin ${ }^{11}$, however whether this is a cause or consequence of placental

164 dysfunction has been unclear. Our mouse model utilizing trophoblast-specific knockout of VE-

165 cadherin exhibits many histopathologic and clinical features of preeclampsia and suggests that

166 loss of VE-cadherin in trophoblasts may be a primary contributor to preeclampsia pathogenesis.

167 Furthermore, trophoblast-specific loss of VE-cadherin may serve as a useful model for studying

168 fetal contributions to preeclampsia. 


\section{References}

1. Velicky P, Knöfler M, Pollheimer J. Function and control of human invasive trophoblast subtypes: Intrinsic vs. maternal control. Cell Adhes Migr. 2016;10(1-2):154-162. doi:10.1080/19336918.2015.1089376

2. Soares MJ, Chakraborty D, Kubota K, Renaud SJ, Karim Rumi MA. Adaptive mechanisms controlling uterine spiral artery remodeling during the establishment of pregnancy. Int J Dev Biol. 2014;58(2-4):247-259. doi:10.1387/ijdb.140083ms

3. Hu D, Cross JC. Ablation of Tpbpa-positive trophoblast precursors leads to defects in maternal spiral artery remodeling in the mouse placenta. Dev Biol. 2011;358(1):231-239. doi:10.1016/j.ydbio.2011.07.036

4. Soares MJ, Varberg KM, Iqbal K. Hemochorial placentation: Development, function, and adaptations. Biol Reprod. 2018;99(1):196-211. doi:10.1093/biolre/ioy049

5. Rai A, Cross JC. Development of the hemochorial maternal vascular spaces in the placenta through endothelial and vasculogenic mimicry. Dev Biol. 2014;387(2):131-141. doi:10.1016/j.ydbio.2014.01.015

6. Lyall F, Robson SC, Bulmer JN. Spiral artery remodeling and trophoblast invasion in preeclampsia and fetal growth restriction relationship to clinical outcome. Hypertension. 2013;62(6):1046-1054. doi:10.1161/HYPERTENSIONAHA.113.01892

7. Roberts JM, Escudero C. The placenta in preeclampsia. Pregnancy Hypertens. 2012;2(2):72-83. doi:10.1016/j.preghy.2012.01.001

8. Nelson AC, Mould AW, Bikoff EK, Robertson EJ. Single-cell RNA-seq reveals cell typespecific transcriptional signatures at the maternal-foetal interface during pregnancy. Nat Commun. 2016;7. doi:10.1038/ncomms11414

9. Zhou Y, Fisher SJ, Janatpour M, et al. Human cytotrophoblasts adopt a vascular phenotype as they differentiate: A strategy for successful endovascular invasion? J Clin Invest. 1997;99(9):2139-2151. doi:10.1172/JCl119387

10. Govindasamy N, Long $\mathrm{H}$, Jeong HW, et al. 3D biomimetic platform reveals the first interactions of the embryo and the maternal blood vessels. Dev Cell. 2021;56(23):32763287.e8. doi:10.1016/j.devcel.2021.10.014

11. Zhou Y, Damsky CH, Fisher SJ. Preeclampsia Is Associated with Failure of Human Cytotrophoblasts to Mimic a Vascular Adhesion Phenotype. J Clin Invest. 1997.

12. Cheng JC, Chang HM, Leung PCK. Transforming growth factor- $\beta 1$ inhibits trophoblast cell invasion by inducing snail-mediated down-regulation of vascular endothelial-cadherin protein. J Biol Chem. 2013;288(46):33181-33192. doi:10.1074/jbc.M113.488866

13. Corada M, Mariotti M, Thurston G, et al. Vascular endothelial-cadherin is an important determinant of microvascular integrity in vivo. Proc Natl Acad Sci U S A. 1999;96(17):9815-9820. doi:10.1073/pnas.96.17.9815

14. Crosby C V., Fleming PA, Argraves WS, et al. VE-cadherin is not required for the formation of nascent blood vessels but acts to prevent their disassembly. Blood. 2005;105(7):2771-2776. doi:10.1182/blood-2004-06-2244

15. Carmeliet $P$, Lampugnani $M G$, Moons $L$, et al. Targeted deficiency or cytosolic truncation of the VE-cadherin gene in mice impairs VEGF-mediated endothelial survival and angiogenesis. Cell. 1999;98(2):147-157. doi:10.1016/S0092-8674(00)81010-7

16. Bulla R, Villa A, Bossi $F$, et al. VE-cadherin is a critical molecule for trophoblastendothelial cell interaction in decidual spiral arteries. Exp Cell Res. 2005;303(1):101-113. doi:10.1016/j.yexcr.2004.09.015

17. Wenzel PL, Leone G. Expression of Cre recombinase in early diploid trophoblast cells of the mouse placenta. genesis. 2007;45(3):129-134. doi:10.1002/dvg.20276

18. López-Tello J, Pérez-García V, Khaira J, et al. Fetal and trophoblast PI3K p110a have distinct roles in regulating resource supply to the growing fetus in mice. Elife. 2019;8:125. doi:10.7554/eLife.45282.001 
19. Galaz J, Romero R, Arenas-Hernandez M, Panaitescu B, Garcia-Flores V, Gomez-Lopez N. A Protocol for Evaluating Vital Signs and Maternal-Fetal Parameters Using HighResolution Ultrasound in Pregnant Mice. STAR Protoc. 2020;1(3):100134. doi:10.1016/j.xpro.2020.100134

20. Hägerling R, Hoppe E, Dierkes C, et al. Distinct roles of VE -cadherin for development and maintenance of specific lymph vessel beds. EMBO J. 2018;37(22). doi:10.15252/embj.201798271

21. Gaengel K, Niaudet C, Hagikura K, et al. The Sphingosine-1-Phosphate Receptor S1PR1 Restricts Sprouting Angiogenesis by Regulating the Interplay between VE-Cadherin and VEGFR2. Dev Cell. 2012;23(3):587-599. doi:10.1016/j.devcel.2012.08.005

22. Gatford KL, Andraweera PH, Roberts CT, Care AS. Animal Models of Preeclampsia: Causes, Consequences, and Interventions. Hypertension. 2020;75(6):1363-1381. doi:10.1161/HYPERTENSIONAHA.119.14598

23. Marshall SA, Hannan NJ, Jelinic M, Nguyen TPH, Girling JE, Parry LJ. Animal models of preeclampsia: translational failings and why. Am J Physiol Integr Comp Physiol. 2018;314(4):R499-R508. doi:10.1152/ajpregu.00355.2017

24. Yang Y, Cha B, Motawe ZY, Srinivasan RS, Scallan JP. VE-Cadherin Is Required for Lymphatic Valve Formation and Maintenance. Cell Rep. 2019;28(9):2397-2412.e4. doi:10.1016/j.celrep.2019.07.072 


\section{Acknowledgments}

We thank members of the Kahn laboratory for thoughtful discussions during the course of these studies, the CDB Microscopy Core for support with microscopy, and the Small Animal Imaging Facility Core for their support with ultrasound imaging. We thank Dr. Jeremy VeenstraVanderweele (Columbia University) and Dr. Gustsavo Leone (Medical University of South Carolina) for kindly providing the Cyp $19^{\text {Cre }}$ mice.

\section{Funding}

This work was supported by NIH grants T32 HL007439 and F30 HL158014 (to DCS), American Heart Association Postdoctoral Fellowship No. 35200213 (to XC), NIH grant T32 HL007971 and American Heart Association Postdoctoral Fellowship No. 836238 (to TCSK), NIH R01 grant HL142905 (to JPS), NIH R01 grant HL145397 (to YY), and NIH R01 grant HL142976 (to MLK).

\section{Author Contributions}

DCS designed and performed most of the mouse and tissue culture experiments. $\mathrm{XC}, \mathrm{MC}, \mathrm{YX}$, SG, and TCSK contributed to mouse studies. JY performed histological studies. SS performed Doppler ultrasounds. AB performed bioinformatics analysis. ML provided animal husbandry support. YY and JPS generated and characterized the Cdh5 conditional allele. DCS and MLK interpreted data and wrote the manuscript.

\section{Competing Interests}

The authors declare no competing financial interests

\section{Data and Materials Availability}

All data and reagents will be made available upon reasonable request. Transgenic mouse lines not available through public repositories are available from Mark Kahn under a material transfer agreement with the University of Pennsylvania. 


\section{Methods}

\section{Generation of Mutant Mice}

Cyp19(Tg)-Cre mice have been previously described ${ }^{17}$ in which the transgene relies on Cre expression under the Cyp19 promoter containing regulatory elements for trophoblast-specific expression, as Cyp19 is not endogenously expressed in trophoblasts. VE-cadherin (Cdh5) floxed mice have been previously described ${ }^{24}$ and were generated with LoxP sites flanking Exons 3 and 4. Mice were bred according to standard protocols and maintained on a mixed background. Male Cdh5 ${ }^{\mathrm{fl/fl}}$ mice were mated to female Cyp19 ${ }^{\mathrm{Cre}} ; \mathrm{Cdh} 5^{\mathrm{fl} /+}$ mice due to the influence of parental inheritance on Cre expression, with maternal inheritance providing the most robust and consistent expression ${ }^{17}$. Mating pairs were set up in the afternoon and vaginal plugs checked in the morning. Presence of a vaginal plug indicated embryonic day (E)0.5. Crenegative $\left(\mathrm{Cdh}^{\mathrm{fl} /+}\right.$ and $\left.\mathrm{Cdh} 5^{\mathrm{fl} / \mathrm{fl}}\right)$ and Cre-positive heterozygous (Cyp19 ${ }^{\mathrm{Cre}}$; Cdh5 $5^{\mathrm{fl} /+}$ ) littermates were used as controls. All procedures were conducted using an approved animal protocol (806811) in accordance with the University of Pennsylvania Institutional Animal Care and Use Committee.

\section{Intrauterine Doppler Ultrasound}

In utero Doppler ultrasound was performed by a trained technician using the VEVO2100 Ultrasound System equipped with the MS-400 transducer (30MHz). E12.5 pregnant mice were lightly anesthetized using $2 \%$ isoflurane. Hair was removed from the abdomen using chemical hair remover (Nair), and the animals were placed on a warming pad. Maternal heart rate and temperature were continuously monitored and consistently within $400-500 \mathrm{bpm}$ and $37^{\circ} \mathrm{C}$. Ultrasound gel was applied to the abdomen and the transducer applied to visualize embryos and placentas using the maternal bladder as an anatomical landmark. Color Doppler was used to visualize the umbilical vessels, and pulse wave (angle of insonation $<60^{\circ}$ ) measurements were made at the point where the umbilical artery inserts into the placenta. From the Doppler waveforms, peak systolic velocity (PSV) and end diastolic velocity (EDV) were measured and used to calculate resistance index $[R I=1-P S V / E D V]$ and pulsatility index $[\mathrm{PI}=(\mathrm{PSV}-$ $\mathrm{EDV}) /$ mean velocity, where mean velocity $=(\mathrm{PSV}+\mathrm{EDV}) / 2]$. Heart rate was calculated as beats per minute by dividing 60 seconds by the systolic+diastolic time.

\section{Histology and Immunofluorescence Staining and Analysis}

Whole mouse embryos or placentas were collected and fixed in $4 \%$ paraformaldehyde (PFA) overnight at $4^{\circ} \mathrm{C}$ prior to dehydration in alcohol and paraffin embedding. Tissue sections 
underwent to dewaxing and rehydration through xylene and ethanol treatment and were then subject to hematoxylin and eosin (H\&E) staining or processed for immunofluorescence. For immunodetection, $10 \mathrm{mM}$ citrate buffer $(\mathrm{pH} 6)$ was used for antigen retrieval, and sections were blocked with $10 \%$ donkey serum in $1 \%$ BSA prior to primary antibody treatment overnight at $4^{\circ} \mathrm{C}$. A list of antibodies can be found below. Fluorescence-conjugated Alexa Fluor secondary antibodies were used (1:500, Invitrogen) according to the primary antibody species and counterstained with DAPI (1:1000). Sections or tissues were mounted on slides with ProLong Gold Antifade reagent. Signals were detected and images collected using a Zeiss LSM 880 confocal microscope and Zeiss Axio Observer 7 widefield microscope. Images were visualized using Image J/FIJI software (NIH).

\section{Quantification of Immunofluorescence Images}

Number of $\mathrm{CK}^{+}$trophoblasts and $\alpha \mathrm{SMA}^{+}$smooth muscle cells were manually counted and divided by total decidual area. Trophoblast invasion depth was measured as the distance the farthest $\mathrm{CK}^{+}$trophoblast was found in the decidua relative to the junctional zone. Percent labyrinth endothelial cell coverage was measured by quantifying Endomucin-positive area as a percentage of total labyrinth area. Trophoblast-endothelial displacement was quantified by measuring the circumference of the vessel lumen and then measuring the length of $\mathrm{CK}^{+}$ trophoblasts in contact with the lumen. Percent displacement was calculated by dividing the trophoblast length by the lumen circumference. All images were analyzed using ImageJ/FIJI software.

\section{Antibodies}

Antibodies for immunofluorescence: Endomucin (1:400, R\&D AF4666 or 1:300, Abcam ab106100), TER119 (1:300, Abcam ab91113), mouse VE-cadherin (1:300, R\&D AF1002), CK8 (1:300, Abcam ab53280 and 1:400, DSHB TROMA-1), aSMA-Cy3 (1:300, Sigma XXX)

\section{Statistical Analysis}

All data are reported as means with $n \geq 3$ independent experiments or mice, and error bars represent standard deviation (SD). Each data point in the figures represents one individual placental or embryo. The explicit number of samples is indicated in the figure legends. No explicit power analyses were used to predetermine sample size, and no randomization was used. No samples were excluded for analysis. Statistical significance was determined using Welch's t-test. Differences between means were considered significant at $P<0.05$. Significant 
bioRxiv preprint doi: https://doi.org/10.1101/2022.02.15.480503; this version posted February 16,2022 . The copyright holder for this preprint (which was not certified by peer review) is the author/funder, who has granted bioRxiv a license to display the preprint in perpetuity. It is made available under aCC-BY 4.0 International license.

differences in expected genotypes was calculated using Fisher's exact test and considered statistically significant at $\mathrm{P}<0.05$. 

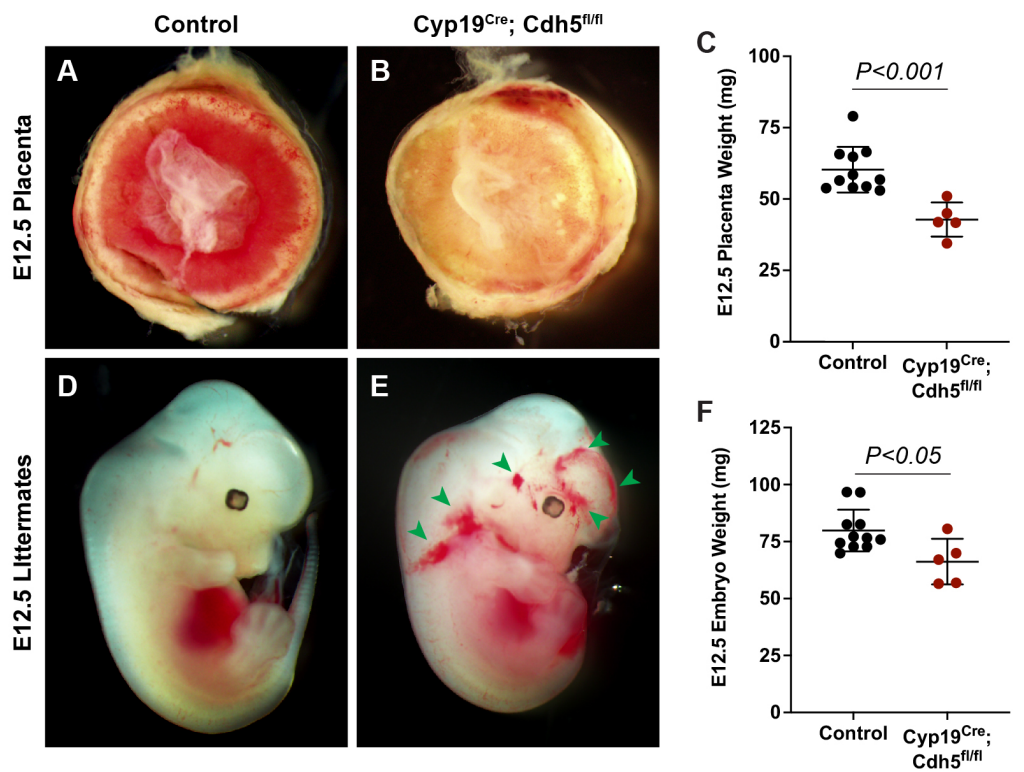

Figure 1. Deletion of VE-cadherin in trophoblasts results in placental and fetal growth restriction. A-C, Gross examination and quantification of E12.5 Control and Cyp19 ${ }^{\mathrm{Cre}}$; Cdh5 $5^{\mathrm{fl} / \mathrm{fl}}$ placentas and weights. Control n=11, Cyp19 $19^{\text {Cre }}$; Cdh5 $5^{\text {fl/fl }} n=5$. D-F, Gross examination and quantification of E12.5 Control and Cyp19 Cre. Cdh5 $5^{\text {fl/fl }}$ embryos and weights. Control n=11, Cyp19 ${ }^{\text {Cre. }}$ Cdh5 $5^{f / f l} n=5$. Statistical analysis was performed using two-tailed, unpaired Welch's ttest. Data are shown as means \pm S.D. 

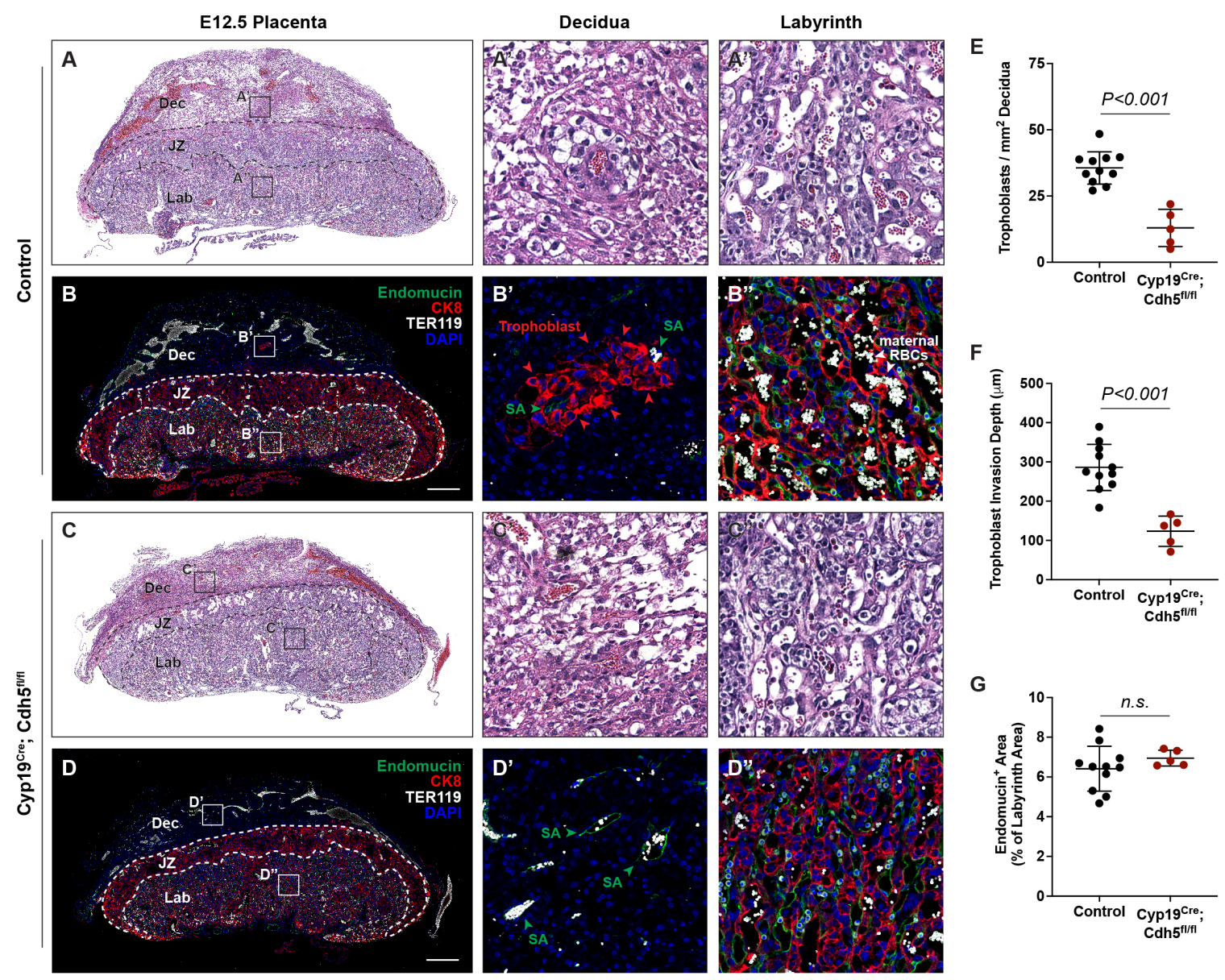

Figure 2. VE-cadherin promotes trophoblast endovascular invasion. A-D, Hematoxylin and eosin (H\&E) staining and immunofluorescence staining for Endomucin (green), CK8 (red), and TER119 (gray) of E12.5 Control (A, B) and Cyp19 ${ }^{\mathrm{Cre}}$; Cdh5 $5^{\mathrm{fl} / f \mathrm{l}}$ (C, D) serial placenta sections. Dotted lines demarcate the different placental regions. Red arrowheads indicate trophoblasts. Green arrowheads indicate spiral arteries. White arrowheads indicate maternal red blood cells (RBCs). Note fewer non-nucleated, maternal TER119+ cells in the labyrinth region of Cyp19Cre; $\mathrm{Cdh}^{\mathrm{fl} / \mathrm{fl}}$ placentas. Boxes on the left correlate with magnified images on the right, and boxes in $\mathrm{H} \& \mathrm{E}$ and immunofluorescence images are of the same region. Scale bars $=500 \mu \mathrm{m}$. Dec (decidua), JZ (junctional zone), Lab (labyrinth). E-G, Quantification of number of trophoblasts in the decidua $(\mathbf{E})$, trophoblast invasion depth $(\mathbf{F})$, and percent labyrinth Endomucin ${ }^{+}$area $(\mathbf{G})$. Control n=11, Cyp19 ${ }^{\text {Cre }}$; Cdh5 ${ }^{\mathrm{fl} / \mathrm{fl}} \mathrm{n}=5$. Statistical analysis was performed using two-tailed, unpaired Welch's t-test. Data are shown as means \pm S.D. 
A

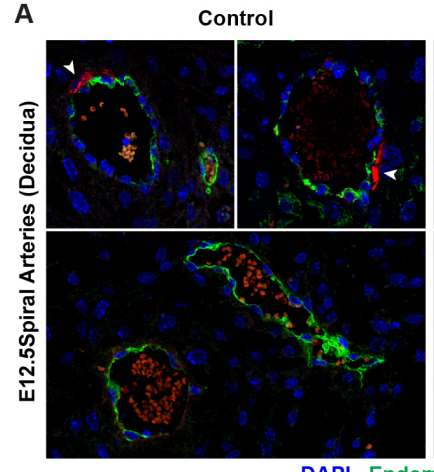

DAPI Endomucin aSMA

C

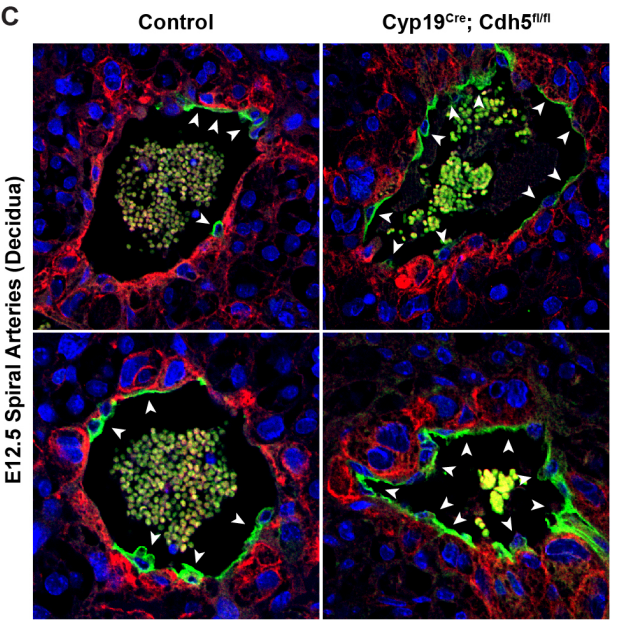

DAPI Endomucin CK8
Cyp19 ${ }^{\text {cre }} ;$ Cdh $^{\text {flifl }}$
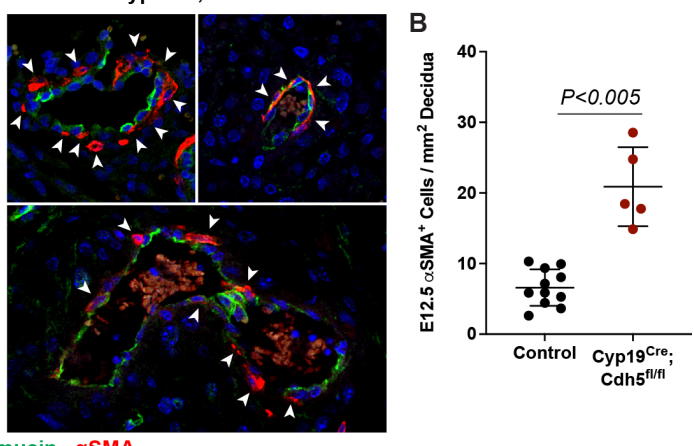

D

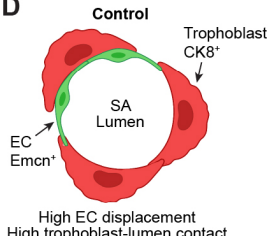

High EC displacement
High trophoblast-lumen contact

E

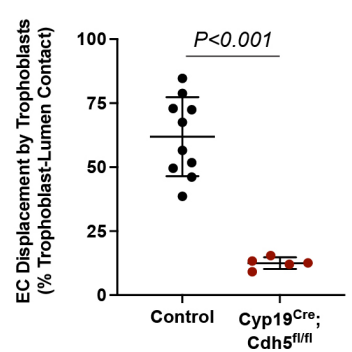

Figure 3. VE-cadherin is required in trophoblasts to remodel spiral arteries and the displace spiral artery endothelium. A, Immunofluorescence staining of E12.5 Control and Cyp $19^{\mathrm{Cre}}$; Cdh5 $5^{\mathrm{fl} / f l}$ placentas for Endomucin (green) and $\alpha \mathrm{SMA}$ (red). White arrowheads indicate $\alpha \mathrm{SMA}^{+}$cells. B, Quantification of $\alpha \mathrm{SMA}^{+}$cells in decidua. Control $n=11$, Cyp19 ${ }^{\text {Cre }}$; Cdh5 ${ }^{\mathrm{fl} / \mathrm{fl}} \mathrm{n}=5$. C, Immunofluorescence staining of E12.5 Control and Cyp19 ${ }^{\mathrm{Cre}}$; Cdh5 $5^{\mathrm{fl} / \mathrm{fl}}$ placentas for Endomucin (green) and CK8 (red). White arrowheads indicate Endomucin ${ }^{+}$spiral artery endothelial cells. D, Schematic demonstrating differences in trophoblast and spiral artery (SA) endothelial cell (EC) contact with the vessel lumen. E, Quantification of the percent trophoblastlumen contact, which was calculated by measuring the circumference of the vessel lumen and then measuring the length of $\mathrm{CK}^{+}$trophoblasts in contact with the lumen. Each point represents the average of at least 3 spiral arteries from an individual placenta. Control $n=10$, Cyp19 ${ }^{\text {Cre. }}$ Cdh5 $5^{\mathrm{fl} / \mathrm{fl}} \mathrm{n}=5$. Statistical analysis was performed using two-tailed, unpaired Welch's ttest. Data are shown as means \pm S.D. 
A

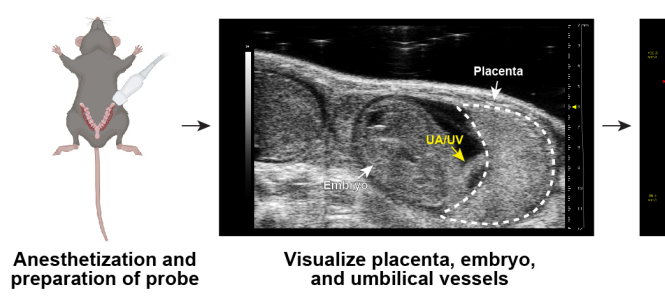
Anesthetization and
preparation of probe

B

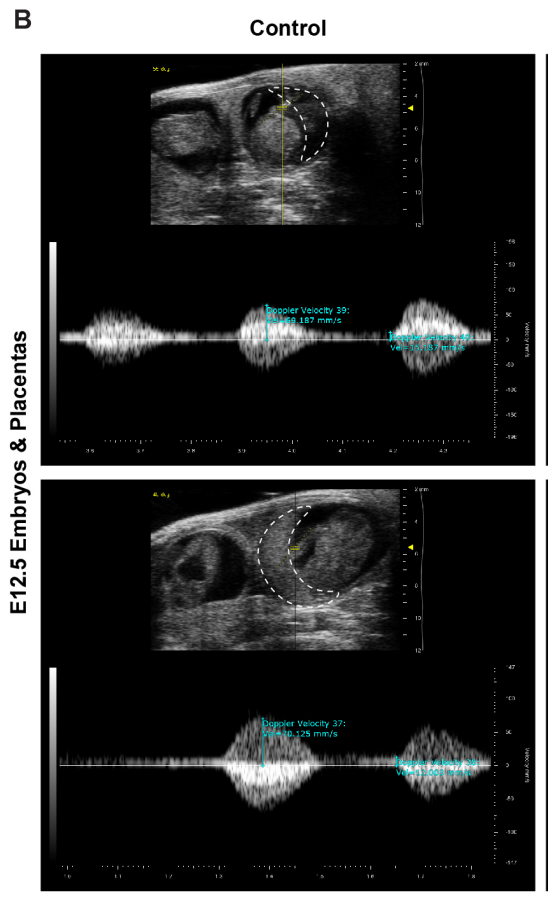

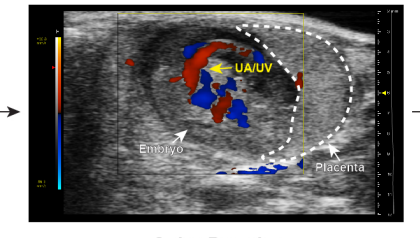

Color Doppler

Cyp19 ${ }^{\text {cre }} ; \mathrm{Cdh}^{\mathrm{fl} / \mathrm{fl}}$
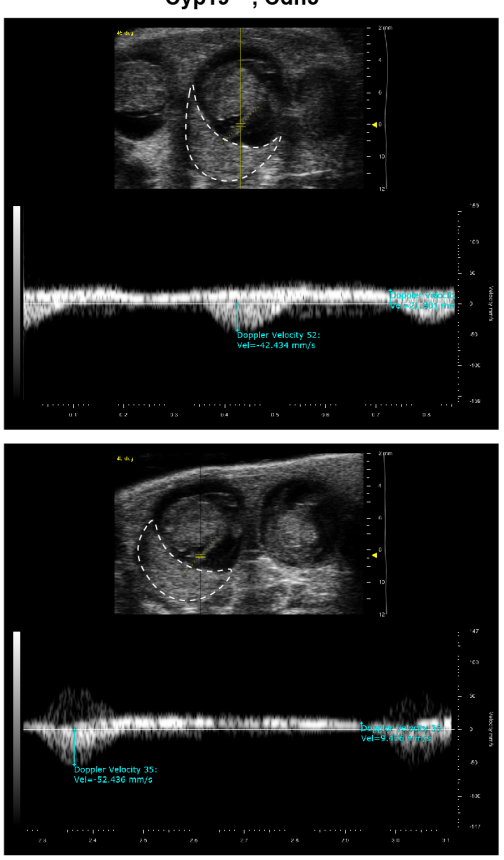

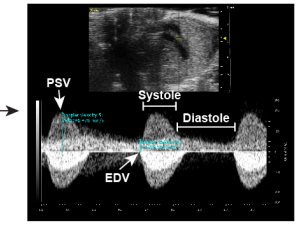

Pulse wave Doppler measurements

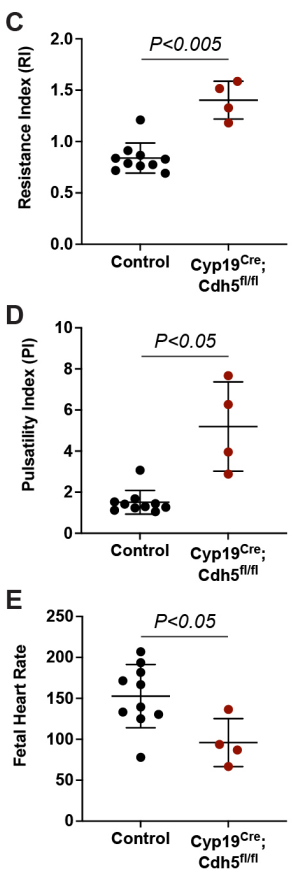

Figure 4. Defects in spiral artery remodeling result in placental insufficiency and fetal distress. A, Schematic of workflow for Doppler ultrasound of pregnant dams. The embryo, UA/UV (yellow arrow), and placenta (dotted white outline) are labeled. B, Representative umbilical artery Doppler waveforms from two control and two Cyp19 ${ }^{\mathrm{Cre}}$; Cdh5 ${ }^{\mathrm{fl} / \mathrm{fl}}$ placentas. The placenta is outlined in a dotted white line. Reversal of end-diastolic flow is evident by the change of directional velocity at the end of diastole compared to peak systole (i.e., negative to positive velocity). C-E, Quantification of resistance index (RI), pulsatility index (PI), and fetal heart rate. PSV (peak systolic velocity), EDV (end diastolic velocity), UA/UV (umbilical artery/umbilical vein). Note that red/blue colors in color Doppler images do not indicate UA/UV, which can only be differentiated based on the Doppler waveform. Control $n=10$, Cyp $19^{\text {Cre; }}$ $\mathrm{Cdh}^{\mathrm{fl} / \mathrm{fl}} \mathrm{n}=4$. Statistical analysis was performed using two-tailed, unpaired Welch's t-test. Data are shown as means \pm S.D. 
bioRxiv preprint doi: https://doi. org/10.1101/2022 $02.15 .480503 \cdot$ this version posted February 16,2022 . The copyright holder for this preprint (which was not certified by peer review) is the author/funder, who has granted bioRxiv a license to display the preprint in perpetuity. It is made available under aCC-BY 4.0 International license.

\begin{tabular}{|c|c|c|c|c|c|c|}
\hline & $\begin{array}{c}\text { Cyp19 } \\
(25 \%)\end{array}$ & $\begin{array}{c}\text { Cyp19 } \text { Cdh5 }^{\mathrm{fl} / \mathrm{fl}} ; \mathbf{C d h 5}^{\mathrm{fl} /+} \\
(25 \%)\end{array}$ & $\begin{array}{c}\mathbf{C d h 5}^{\mathrm{fl} / \mathrm{fl}} \\
(25 \%)\end{array}$ & $\begin{array}{c}\text { Cdh5 }^{\mathrm{fl} /+} \\
(25 \%)\end{array}$ & $\begin{array}{c}\text { Total } \\
(100 \%)\end{array}$ & $\begin{array}{c}\text { Fisher Exact } \\
\text { Test }\end{array}$ \\
\hline E10.5-E12.5 & $12^{*}(26.7 \%)$ & $12(26.7 \%)$ & $9(20 \%)$ & $12(26.7 \%)$ & $45(100 \%)$ & $P=0.7667$ \\
\hline E14.5-E16.5 & $1(2.9 \%)$ & $13(38.2 \%)$ & $12(35.3 \%)$ & $8(23.5 \%)$ & $34(100 \%)$ & $P=0.0033$ \\
\hline P21 & $1(2.2 \%)$ & $11(24.4 \%)$ & $15(33.3 \%)$ & $18(40.0 \%)$ & $45(100 \%)$ & $P=0.0333$ \\
\hline
\end{tabular}

Figure 1-Table Supplement 1. Decreased survival of Cyp19 ${ }^{\mathrm{Cre}}$; Cdh5 ${ }^{\mathrm{fl} / \mathrm{fl}}$ mutants in late gestation. Cdh $5^{\mathrm{fl} / \mathrm{fl}}$ male mice were crossed with Cyp $19^{\mathrm{Cre}} ; \mathrm{Cdh} 5^{\mathrm{fl} /+}$ females to generate litters with mixed genotypes. The expected percentage is listed under the genotype label. The observed number of each genotype is shown with the corresponding percentage given in parentheses. P-values were calculated using Fisher's exact test at stages E10.5-12.5 (pooled), E14.5-16.5 (pooled), and P21. E designates embryonic day and P designates postnatal day. *One embryo at E12.5 was found to be dead. 

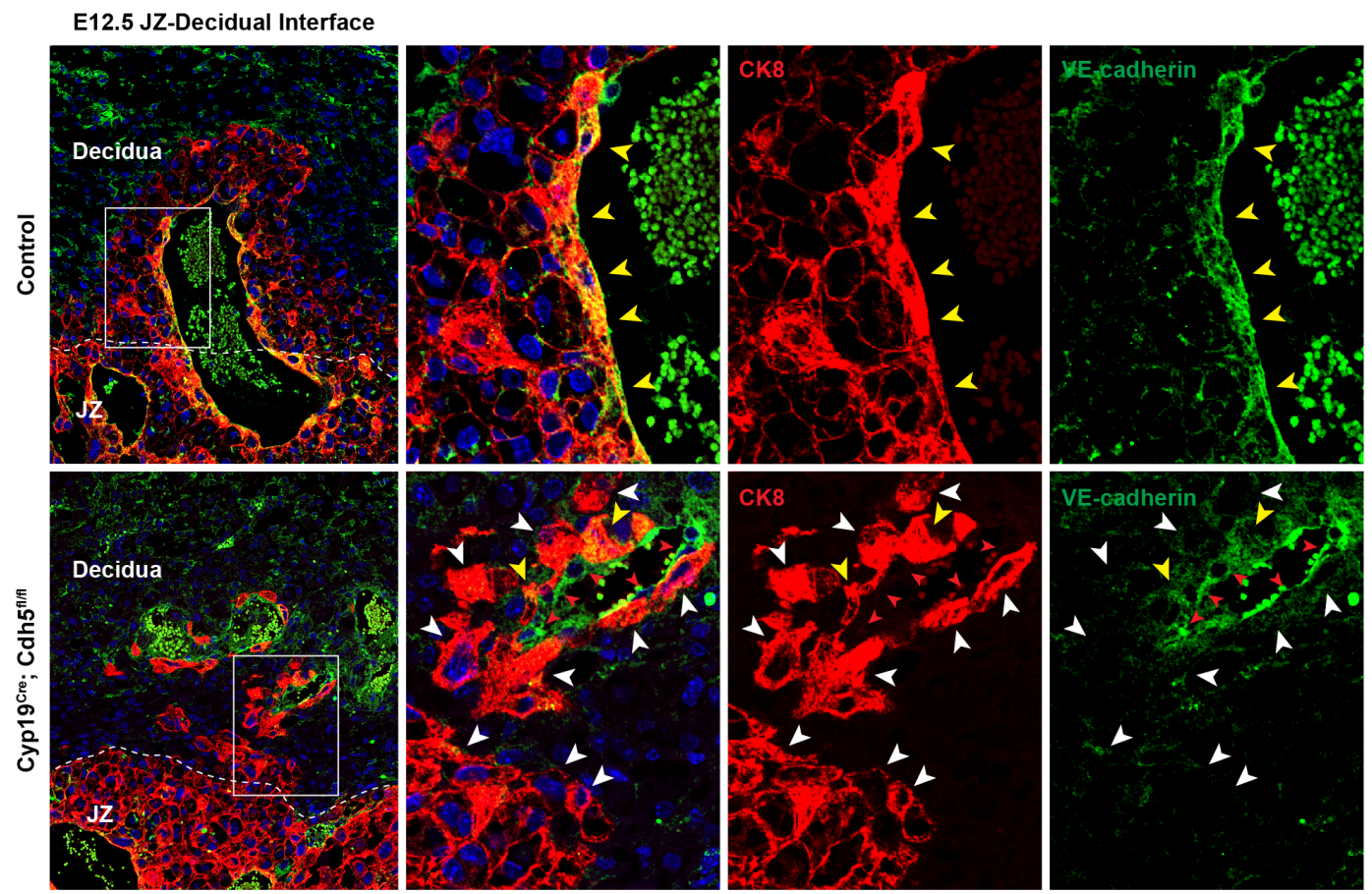

DAPI VE-cadherin CK8

Figure 1-Figure Supplement 1. Deletion of VE-cadherin in Cyp19 ${ }^{\mathrm{Cre}}$; Cdh5 ${ }^{\mathrm{fl} / \mathrm{fl}}$ placentas. Immunofluorescence staining of E12.5 Control and Cyp19 ${ }^{\mathrm{Cre}}$; Cdh5 $5^{\mathrm{fl} / \mathrm{fl}}$ placentas for VE-cadherin

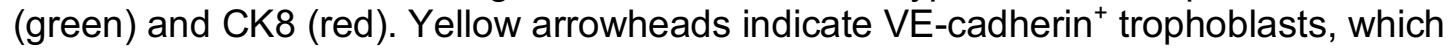
predominantly line remodeled spiral arteries. White arrowheads indicate VE-cadherin ${ }^{-}$ trophoblasts. Red arrowheads indicate spiral artery ECs, which are VE-cadherin ${ }^{+} \mathrm{CK}^{-}$. Note that there are a few VE-cadherin ${ }^{+}$trophoblasts in Cyp19 ${ }^{\text {Cre }}$; Cdh5 $5^{\text {fl/fl }}$ placentas, however VEcadherin is deleted from the majority of trophoblasts. The layer of VE-cadherin ${ }^{+} \mathrm{CK}^{-}$cells (red arrowheads) in the Cyp $19^{\mathrm{Cre}}$; Cdh $5^{\mathrm{fl} / \mathrm{ll}}$ placentas are spiral artery endothelial cells that have not been displaced by trophoblasts. Dotted white lines demarcate the decidua and junctional zone $(\mathrm{JZ})$. 

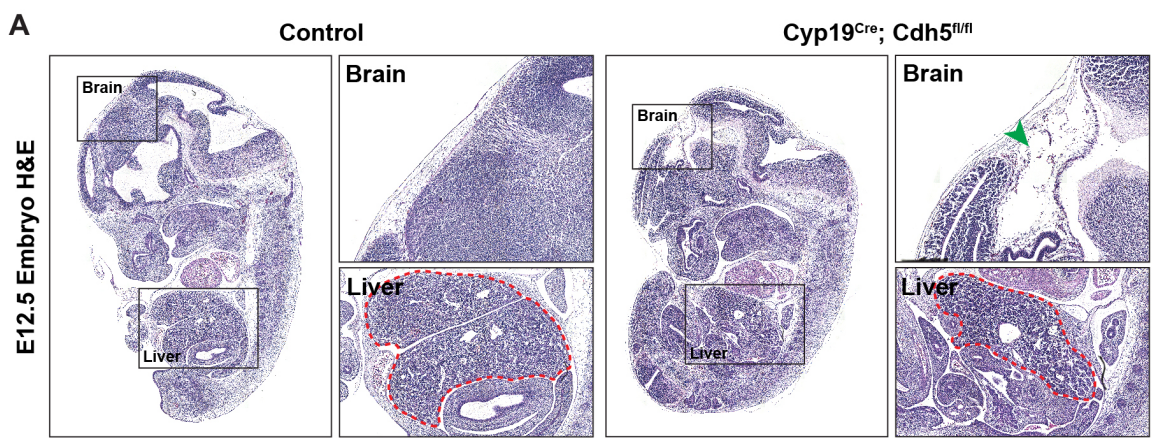

B
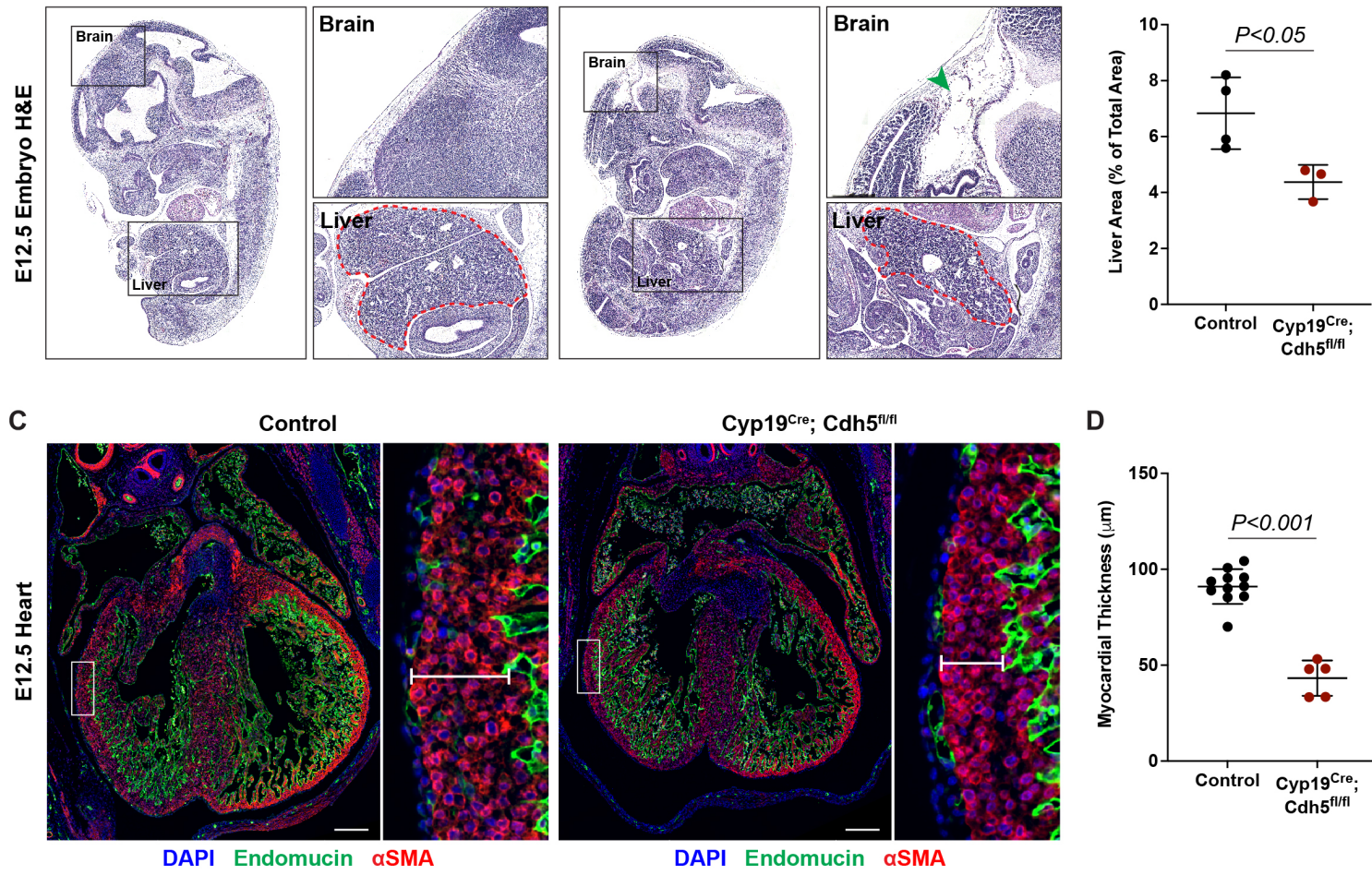

D

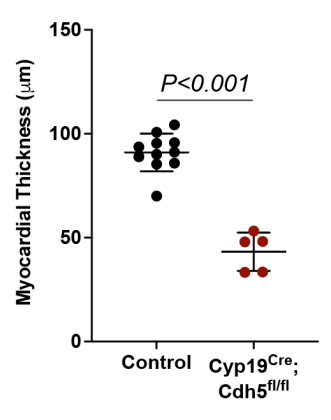

Figure 1-Figure Supplement 2. Loss of trophoblast VE-cadherin causes defects in brain, liver, and heart development. A, Sagittal H\&E sections from E12.5 control and Cyp19 Cre; Cdh5 ${ }^{f / f l}$ embryos. Boxes show magnified regions of the brain, heart, and liver. Note the thinness of the myocardium in Cyp19 ${ }^{\mathrm{Cre}}$; Cdh5 $5^{\mathrm{fl} / \mathrm{fl}}$ embryos. Dotted red line outlines the liver contour. Green arrowhead points to tissue degradation in the brains of Cyp19 ${ }^{\mathrm{Cre}}$; Cdh $5^{\mathrm{fl} / \mathrm{fl}}$ embryos. B, Quantification of the liver area as a percent of total embryo area. Control n=4, Cyp19 ${ }^{\mathrm{Cre}}$; Cdh5 $5^{\mathrm{fl} / \mathrm{fl}}$ n=3. C, D, Immunofluorescence staining of E12.5 control and Cyp19 ${ }^{\mathrm{Cre}}$; Cdh5 $5^{\mathrm{fl} / \mathrm{fl}}$ embryonic hearts for Endomucin (green) and $\alpha \mathrm{SMA}$ (red). Insets demonstrate myocardial thinning and quantified in (D). Control $n=11$, Cyp19 ${ }^{\text {Cre }}$; Cdh $^{\text {fl/fl }} n=5$. Scale bars $=200 \mu m$. Statistical analysis was performed using two-tailed, unpaired Welch's t-test. Data are shown as means \pm S.D. 